\title{
Anti-FCRH5/CD3 BiTE Antibody BFCR4350A
}

National Cancer Institute

\section{Source}

National Cancer Institute. Anti-FCRH5/CD3 BiTE Antibody BFCR4350A. NCI Thesaurus.

Code C139549.

A proprietary recombinant bispecific $\mathrm{T}$-cell engager (BiT E) antibody composed of two single-chain variable fragments (scFv), one directed against the tumor-associated antigen (TAA) Fc receptor-like protein 5 (FCRH5; CD307; FCRL5; IRTA2; BXMAS1) and one that is directed against the CD3 antigen found on T-lymphocytes, with potential immunostimulating and antineoplastic activities. Upon administration of anti-FCRH5/CD3 BiT E antibody BFCR4350A, the bispecific antibody binds to both the CD3 antigen on cytotoxic T-lymphocytes (CTLs) and FCRH5 found on FCRH5-expressing tumor cells. This activates and crosslinks CT Ls with FCRH5-expressing tumor cells, which results in the CT L-mediated cell death of FCRH5-expressing tumor cells. FCRH5, an immune receptor translocation-associated protein/Fc receptor homolog (IRTA/FCRH) family member and a B-cell lineage marker, is overexpressed on myeloma cells. 